ARTICLE HISTORY: Received: September 20, 2021 Accepted: November 13, 2021 Published: November 19, 2021

\title{
MODELING AND INVESTIGATION OF THE REGULATOR WORKING IN THE POINTWISE SLIDING MODE
}

Gasimov Asif Yusif-oqlu, Mammedova Afaq Tofiq

Azerbaijan Technical University.

AZ1073 Azerbaijan,pr.Dzavida 25,Baku.

\begin{abstract}
The main disadvantage of the classic sliding mode is that the control signal is made of high frequency variations. In the recommended pointwisesliding mode the frequency of the variations decreases, since the control signal makes jumps at the isolated points. At the same time, the trajectory remains in a small neighborhood of the plane of switching. In the finish mode, the trajectories that start over the sliding plane reach simultaneously the equilibrium point. It is possible to assume that these regulators will expand the implementation scope of the sliding (quazisliding) modes.
\end{abstract}

Keywords: finite mode, pointwise sliding mode, Regulator

Retention of the robust property of the system and simultaneously reducing the frequency of the control signal can provide the expansion of the application of the regimes. Considering this in the work the establishment and investigation of the "pointwise sliding mode" is discussed.

The pointwise mode differs from the classic sliding mode by the fact that here the jumps of the control signal on the line $s=0$ occur at the isolated points. As the time interval between these points can be adjusted, it is possible to get the following modes:

- finite mode. In this mode all trajectories are reached to the stability point at the finite time interval as a result of single chage of the control;

- Pointwise sliding mode. In this mode, the control signal is exposed to finite jumps; it is possible to increase the frequency of vibrations (as in the ordinary mode) by changing the setting parameters [3].

- Ordinary sliding mode. In this mode, the frequency of the variation of the control signals is closer to infinity;

- Change mode. In this mode, the changes in the value and sign of the control signal occur when the phase trajectory exceeds the line $s=0$ and the ordinate axis $\varepsilon=0$. [4].

Fig. 1, a-c shows the change of control signal in the appropriate modes and phase portraits.
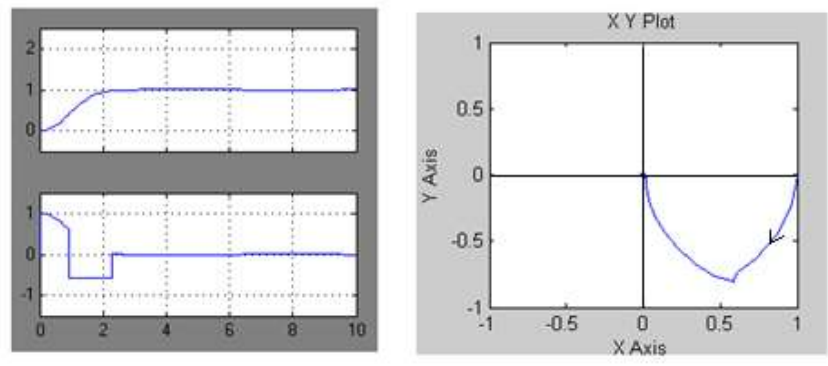

a)
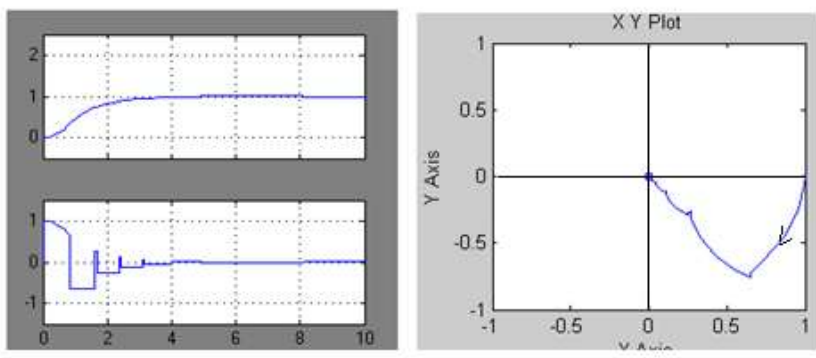

b) 

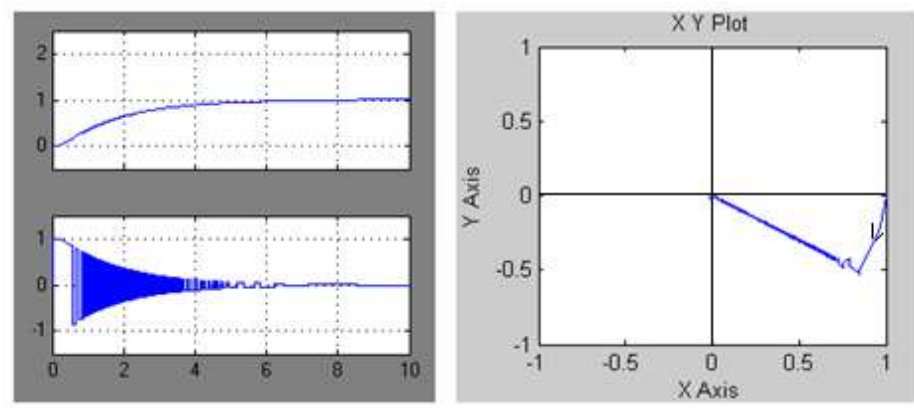

c)

Fig. 1. Characteristics of the exusting modes a) finite mode; b) pointwise sliding mode;c) ordinary mode

The regulation low for the second order linear objects is as follows

$$
u=\left\{\begin{array}{l}
-\alpha \varepsilon \text { if } \sigma>0 \\
\beta \varepsilon_{g}=\text { constəaif } \sigma<0 .
\end{array}\right.
$$

Here $\sigma=s \varepsilon$ is the variation function; $s=c \varepsilon+\varepsilon=0$ is the variation line; $\alpha_{s} \beta>0$ are the the regulator strength coefficients; $\varepsilon_{g} \in\{\varepsilon s=0\}$.The setting parameters $\beta, c$ are defined by the spesific methodology computer simulaton.

Fig. 2 shows the Simulink scheme (a), dynamic (b) and static (c) characteristics of the regulator operating in the pointwise sliding mode.

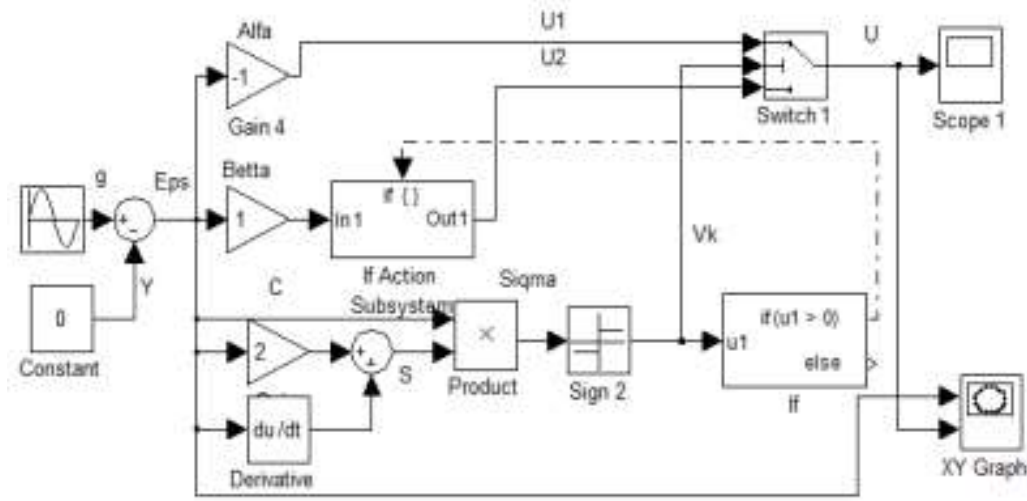

a)
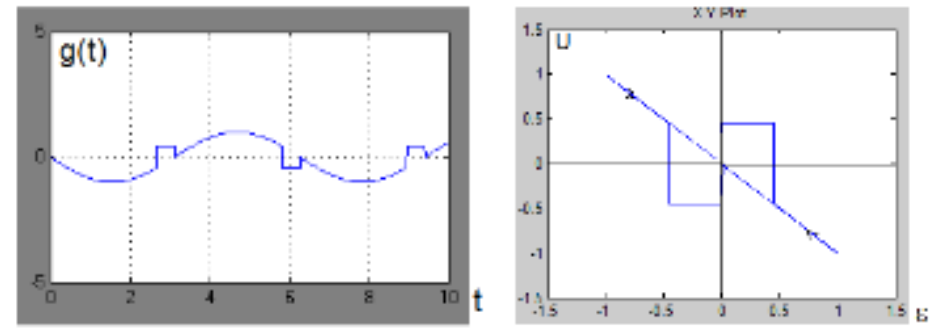

b) c)

Fig. 2. Simulink scheme (a), dynamic (b) and static (c) characteristics for the regulator working in the pointwise sliding mode

This regulator is a robust variable structure regulator that allows reducing the frequency of the control signal in the known sliding mode [1,2].

Different from the classical methods in the pointwise sliding mode, while reducing the frequency of the control signal when the object's parameters and external inluences vary in a wide interval it becomes possible to provide movement (robustness) of the system's together with close neighbourhood of the of the system variation line. At the same time, it was prevented the occuring the autovibtations in the smallneighbourhood of the stability point. 


\section{REFERENCES}

1.G.A. Rustamov. Robust control system with increased potential. News of Tomsk Polytechnic University, Vol.324, No.5, 2015, pp.13-19.

2.V.I. Utkin. Sliding Modes in Optimization and Control Problems. SpringerVerlag, NewYork, 1992.

3.S.V. Yemelyanov, S.K.Korovin. New types of feedback: Control under uncertainty. Moscow, Nauka, 1997, $352 \mathrm{p}$.

4. Gasimov A. Y., Farkhadov V. G., Yolchuyev I. A. Synthesis and implementation of controllers operating in point slidint mode. Materials of the IV International scientific-tehnical conference "Computer modelling and optimization of complex systems". Dnepr, Ukraine, 2018, pp. 149-152. 